\title{
Study on Transient Electromagnetic Response of High Resistivity Goafs and Its Application
}

\author{
Maofei $\mathrm{Li}^{1}$, Shucai Liu ${ }^{1}$, Benyu Su${ }^{1}$, Yongsheng $\mathrm{Ma}^{2}$, Qilong Sun ${ }^{2}$ \\ ${ }^{I}$ The School of Resource and Geoscience, China University of Mine and Technology, \\ Xuzhou 221116, Jiangsu Province, China \\ 2The Key Laboratory of Unconventional Petroleum geology, CGS \\ subenyu@cumt.edu.cn
}

\begin{abstract}
Based on the characteristics of mine goafs, the geo-electrical models are established. In order to study the response characteristics of goaf by transient electromagnetic method, we have performed three-dimensional numerical simulations in three cases, geo-electrical model of mine geology without goaf, geo-electrical model of mine geology with low resistivity goaf and geo-electrical model of mine geology with high resistivity goaf. Moreover, the physical experiments have also been performed for the above three cases. Furthermore, we compare numeral simulation results and physical experiment results. Finally, we obtain the conclusion that although the response of high resistivity goafs is weaker than that of low resistivity, its response characters still can indicate the existing of goaf. Hence, above work actually signifies that shallow high resistivity goafs can be detected in a reliable way by transient electromagnetic method.
\end{abstract}

Index Terms-Transient electromagnetic method; Time-Depth conversion; High resistance goafs; Physical modelling.

\section{INTRODUCTION}

Because of continuous coal mining in China, the problems of coal mine goafs is becoming more and more serious and it has brought a series of social problems [1]. However, the most serious goafs are in shallow earth and without collapse which are major danger potential for the safety production of coal mine. Especially, the goafs were formed by early private coal mines. Furthermore, these goafs are shallow burial and scattered distribution, hence it is very difficult to confirm the boundaries and locations. Usually, they will bring great damage to later coal mine and ground infrastructure construction [2]-[4].

Generally speaking, the goafs are mainly classified into the high resistivity goafs unfilled with water and the low resistance goafs filled with water. The electrical properties of two kinds of goafs are quite different from the surrounding rock [5]-[7]. Therefore, the electromagnetic methods can effectively detect goafs by the resistivity

Manuscript received 26 May, 2018; accepted 11 November, 2018.

This research was funded by National Natural Science Foundation (No. 41674133) and sub-item Major National Science and Technology (No. 2016YFC060110204). difference between goaf and souring rock of the earth. In recent years, geophysical workers in China have performed a lot of researches on the exploration of goafs, but the jobs are mainly focused on the low resistivity goafs filled with water [8]-[10]. The reason is that the responses of high resistivity target bodies detected by electromagnetic methods are not clearly observed. However, identified high resistivity goafs unfilled with water is the major challenge to construction of ground infrastructure in the future. So it is necessary for the detection of high resistivity goafs. Compared with other geophysical method, Transient Electromagnetic Method (TEM) has the following traits, convenient operating equipment, high efficiency and effectiveness. Based on above advances of transient electromagnetic method, we try to detect high resistance goaf by transient electromagnetic method.

\section{NUMERICAL CALCULATION}

\section{A. $1 D$ Case of Numerical Simulation}

The rectangular as transmitter is employed to perform 1D numerical simulation of transient electromagnetic method. It can be thought to be formed by finite small vertical magnetic dipoles [11]. Hence, first we compute the electromagnetic response of the model in the frequency domain [12], and then we convert it from frequency domain to the time domain by Gaver-Stehfest (G-S) transform method [13]. For the numerical simulation, we choose two earth models formed by high resistivity layers and low resistivity layers and the parameters of the models as shown in Table I and Table II, respectively. From Table I and Table II, we can see that we perform the numerical simulation by only changing the thickness of the first layer. Four side lengths of transmitter rectangular are all 600 meter and the current is $10 \mathrm{~A}$. Besides, the measurement point is central of the rectangular.

TABLE I. THE PARAMETERS OF LOW RESISTIVITY.

\begin{tabular}{|c|c|c|}
\hline & Resistivity $(\mathbf{\Omega} \cdot \mathbf{m})$ & Thickness $(\mathbf{m})$ \\
\hline First layer & 100 & $\begin{array}{c}10,30,50,70,90, \\
110,150\end{array}$ \\
\hline Second layer & 0.1 & 10 \\
\hline Third layer & 100 & \\
\hline
\end{tabular}


TABLE II. THE PARAMETERS OF HIGH RESISTIVITY.

\begin{tabular}{|c|c|c|}
\hline & Resistivity $\mathbf{( \mathbf { \Omega } \cdot \mathbf { m } )}$ & Thickness $(\mathbf{m})$ \\
\hline First layer & 100 & $\begin{array}{c}1,10,30,50,70,90, \\
110,150\end{array}$ \\
\hline Second layer & 100000 & 10 \\
\hline Third layer & 100 & \\
\hline
\end{tabular}

Figure 1(a) is the numerical simulation results of model with the low resistivity goaf and Fig. 1(b) is the numerical simulation results of model with the high resistivity goaf. Compared with the Fig. 1(a) and Fig. 1(b), the electromagnetic response of model with low resistivity goaf are more sensitivity than that of model with high resistivity. However, we can improve the sensitivity of the model with high resistivity goaf by subtracting background field [14].

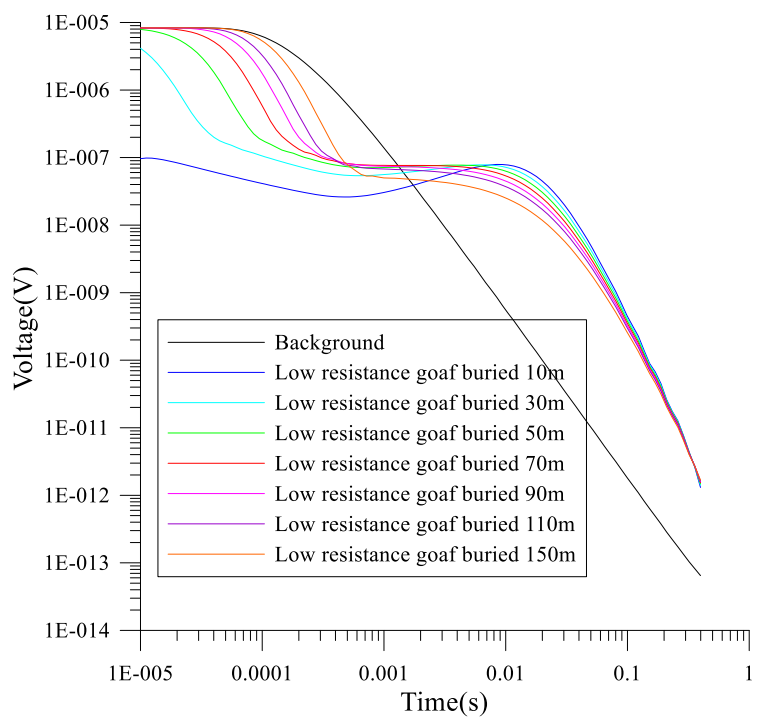

(a)

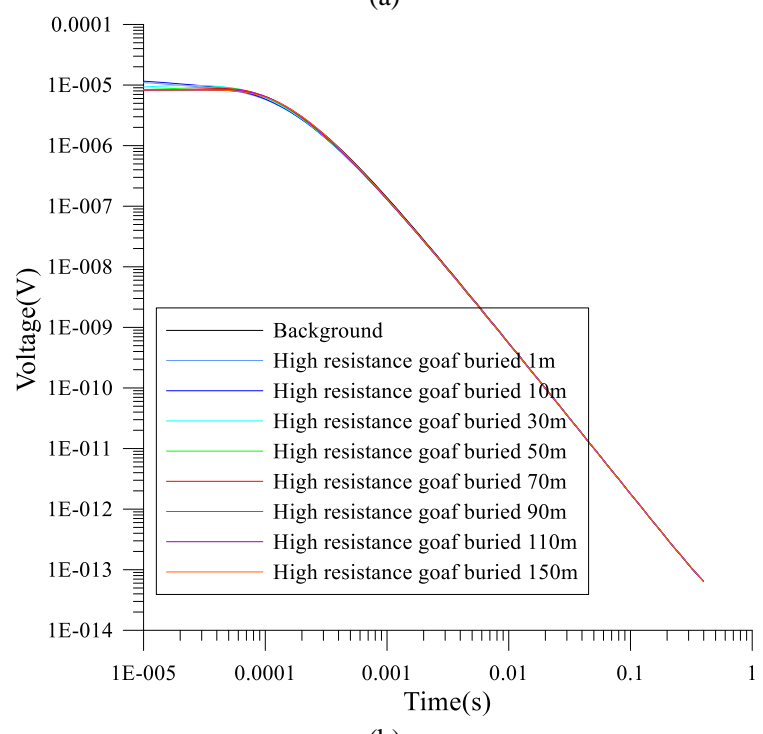

(b)

Fig. 1. Voltage curve as a function of time: a) voltage curve of model with low resistivity goaf; (b) voltage curve of model with high resistivity.

Figure 2 shows the curves of the difference between models electromagnetic response with goaf present and the background field. In particular, Fig. 2(a) corresponds to the model of low resistivity goaf which shows that the positive peak appears in early period. In an opposite way, Fig. 2(b) corresponds to the model of high resistivity goaf which shows that the negative peak appears in early period and the positive peak appears in the late period. In particular, when the thickness of the first layer is more than $110 \mathrm{~m}$, the difference between model with high resistivity goaf and background field is so small that we cannot recognize the existence of the goaf, especially in the case of presence of noise.

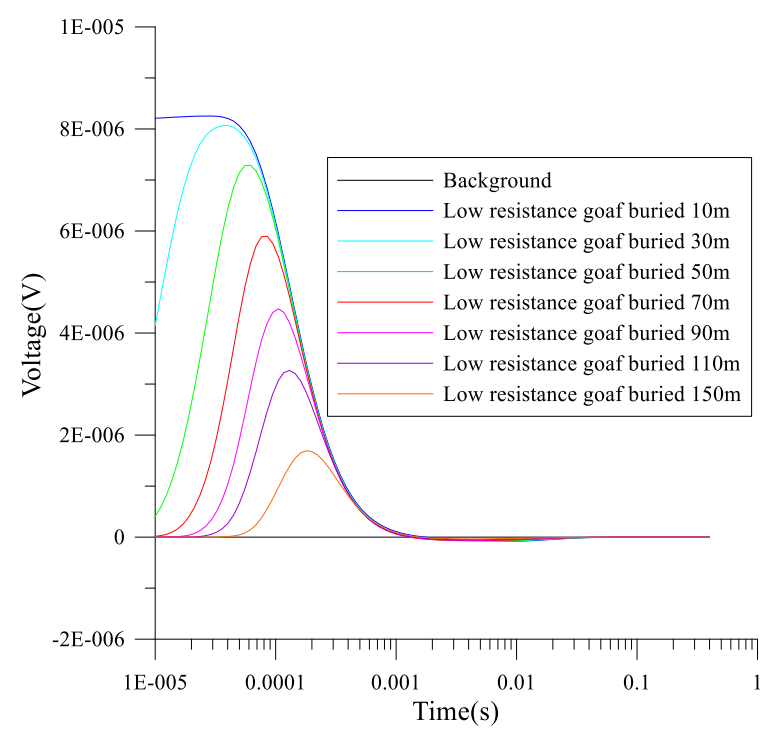

(a)

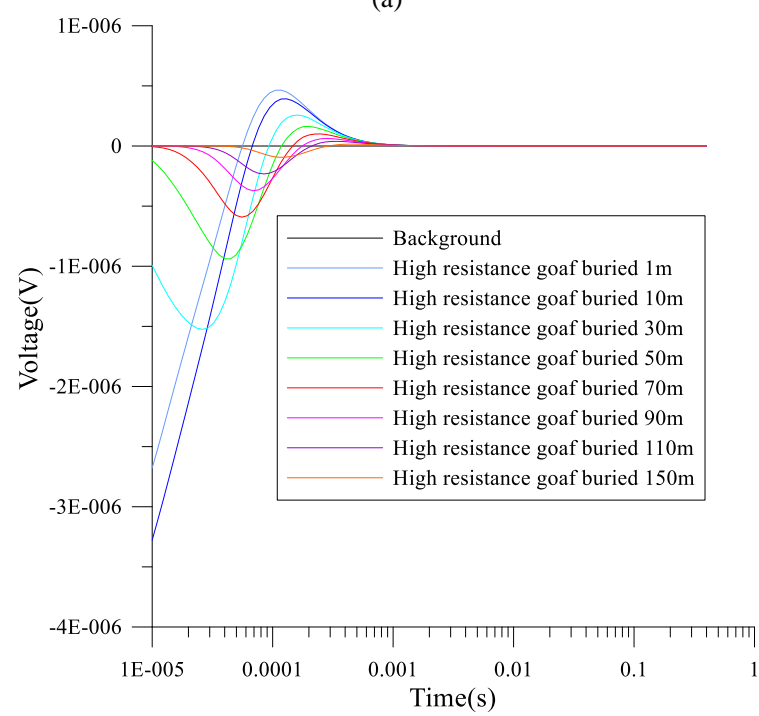

(b)

Fig. 2. Difference curves between response of model and background field: a) Difference curves between response of model with low resistivity goaf and background field; b) Difference curves between response of model with high resistivity goaf and background field.

\section{B. $3 D$ Case of Numerical Simulation}

Based on the 1D modelling results, we have performed the 3D numerical simulation in the following parts. According to the characteristic of goafs, two 3D models are designed. A high resistivity goaf unfilled with water and a low resistivity goaf filled with water are in the coal seam, as shown in Fig. 3, respectively. Volumes of two goafs are all $100 \mathrm{~m} \times 100 \mathrm{~m} \times 20 \mathrm{~m}$. Besides, the size of transmitting coil is $600 \mathrm{~m} \times 600 \mathrm{~m}$, the transmitter current is $10 \mathrm{~A}$ and receivers are set in the centre of the coil.

The response curves of transient electromagnetic method of above models are shown in Fig. 4. Compared with background response, we cannot detect any anomalous response behaviour from the curves, as shown in Fig. 4. However, useful results are found after subtracting the 
background response from the two curves, as shown in Fig. 5.

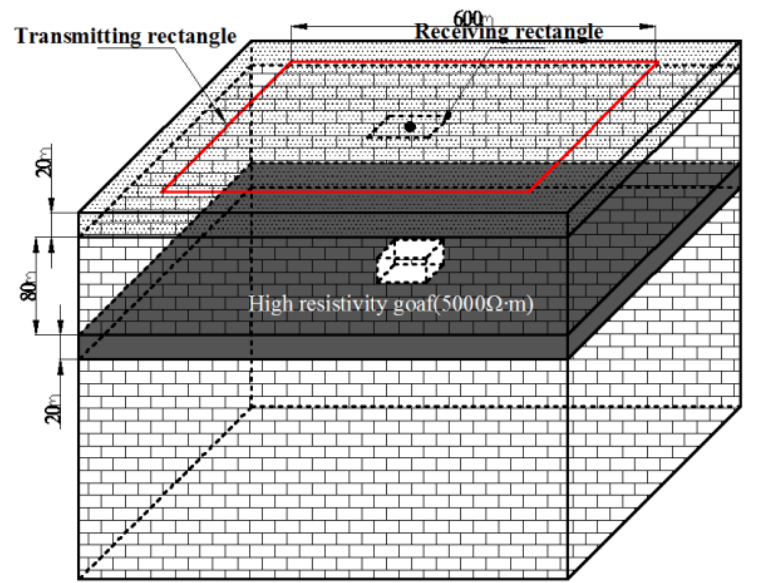

(a)

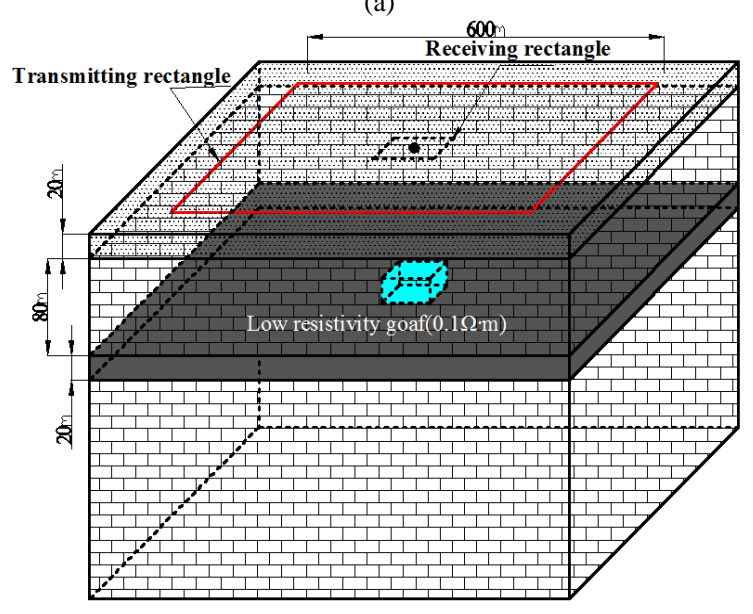

(b)

Fig. 3. The models of different goaf.

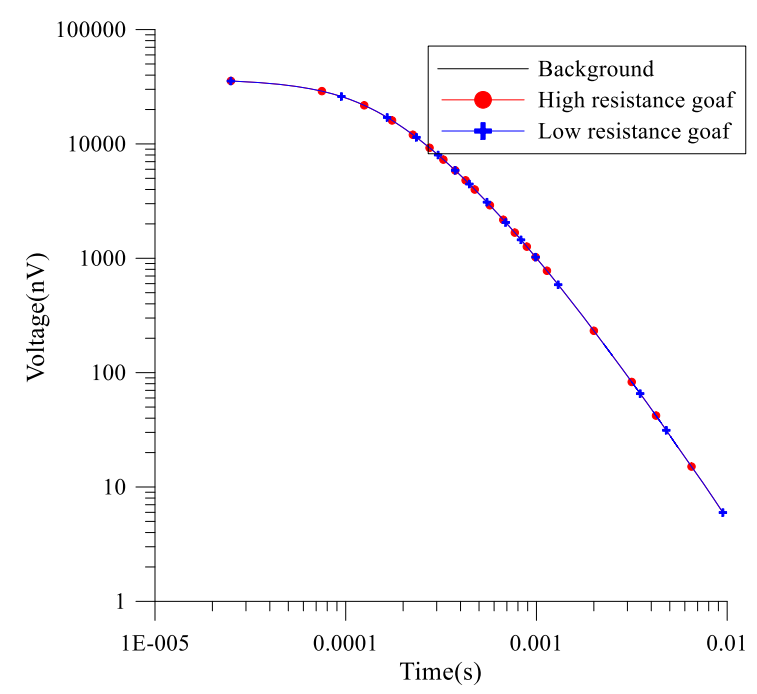

Fig. 4. Response curve of TEM.

This figure shows that the response value of high resistivity goaf is less than the response value of low resistivity goaf. Furthermore, in the early stage, the response value of high resistivity goaf is negative. However, the curve becomes positive in the late stage. For low resistivity goaf, the response characteristic is opposite to the high resistance goaf and much higher in absolute value.

The detected results, as shown in Fig. 4, are usually converted into apparent resistivity and detected depth. Actually, electrical voltage value described in the vertical axis is converted into apparent resistivity by the (1), and the time value is changed to detected depth by the (3) [15]

$$
\rho_{s}^{L}=\frac{\mu}{4 \pi}\left(\frac{2 \mu M}{5 V / S_{r}}\right)^{2 / 3} t^{-5 / 3} .
$$

In the above formula, $\mu$ is magnetic permeability, $\mu_{0}=4 \pi \times 10^{-7} H / m$ for the free air; $M=I L^{2}$, where transmitter electrical current is $I(\mathrm{~A})$ and $L$ is the side length of transmitting rectangle coil $(\mathrm{m}) ; V$ is the induced electromotive force $(\mathrm{V}) ; S_{r}$ is the effective area of rectangle receiving coil $\left(\mathrm{m}^{2}\right)$, and $\mathrm{t}$ is measurement time.

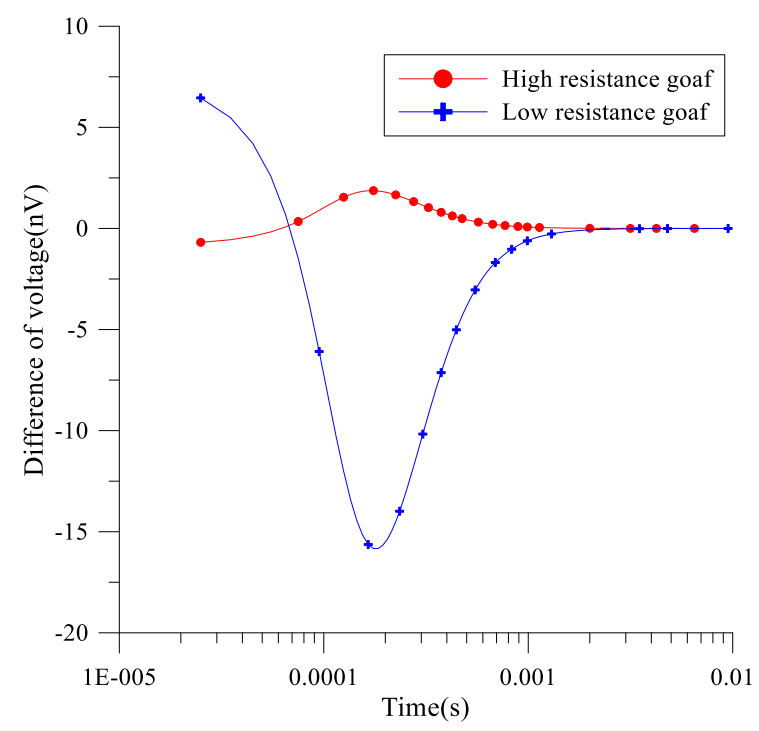

Fig. 5. Response difference curve of TEM.

According to the research of Jiang B. Y. (1998) [16], the equation for the velocity of propagation of the electromagnetic (EM) waves in the earth is given by the following formula

$$
V_{i}=\alpha \frac{\sqrt{\gamma}}{\sigma_{i} \mu a}\left\lfloor C_{1}+\left(C_{1}^{2}+2\right)^{1 / 2}+\left(1+\frac{C_{1}}{\left(C_{1}^{2}+2\right)^{1 / 2}}\right) \gamma C_{2}\right\rfloor,
$$

where:

$$
\begin{gathered}
C_{1}(\gamma)=\frac{3 \sqrt{\pi}}{4}\left[1-\frac{\gamma}{4}-\sum_{k=2}^{\infty} \frac{(2 k-3) ! !}{k !(k+1) !}\left(\frac{\gamma}{2}\right)^{k}\right], \\
C_{2}(\gamma)=\frac{3 \sqrt{\pi}}{4} \sum_{k=0}^{\infty} \frac{(2 k-1) ! !}{k !(k+1) !}\left(\frac{\gamma}{2}\right)^{k}, \\
\gamma=\sigma_{i} \mu a^{2} / 4 t_{i},
\end{gathered}
$$

here $\sigma_{i}$ is the reciprocal of apparent resistivity at moment $t_{i} ; \alpha$ is the coefficient of proportionality. Then the depth of the $t_{i}$ can be calculated by (6)

$$
h_{i}=V_{i} \times t_{i} .
$$




\section{PHYSICAL MODELLING}

An existing model which shown in Fig. 6 is used to do physical experiments the model includes three parts from the roof to the floor. Here, both of the top part and bottom part are Quaternary clay which resistivity is low. Besides, the middle part is filled with high resistivity coal seam. A transient electromagnetic equipment named "Terra-TEM" is used to measure data. The line with red colour is measured path shown in Fig. 6(a), and the interval between two neighbour measuring points is $50 \mathrm{~cm}$. The size of rectangle transmitting coil is $50 \mathrm{~cm} \times 50 \mathrm{~cm}$ and the number of turns is 5. The size of rectangle receiving coil is $20 \mathrm{~cm} \times 20 \mathrm{~cm}$ and the number of turns is 20 .

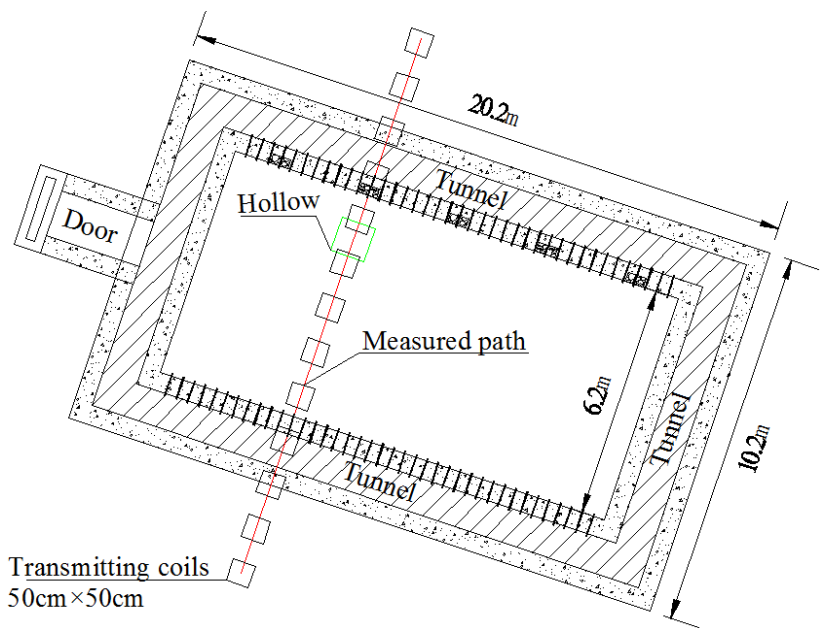

(a)

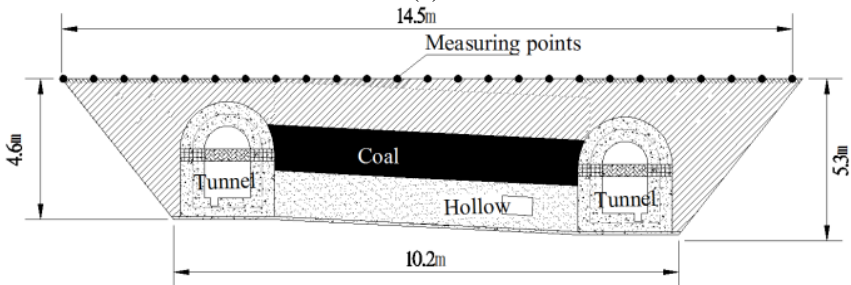

(b)

Fig. 6. Physical model: a) Top view of physical model; b) Side view of physical model.

According to the physical model mentioned above, the induced voltage is measured, as shown at Fig. 7 which is composed by voltage measured by different time.

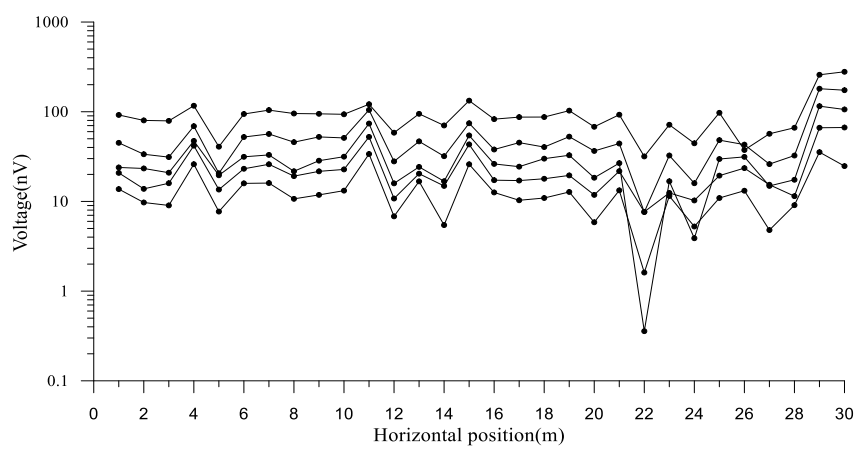

Fig. 7. Measured induced voltage.

Usually, the value of early time is larger than that of late time. And then, based on measured voltage and measuring time, the apparent resistivity and time-depth conversion of (1), (2) and (6) are adopted, and the apparent resistivity profile is drawn, as shown in Fig. 8 and from which we can find high resistivity anomalous geological bodies which are the two tunnels and one cavity.

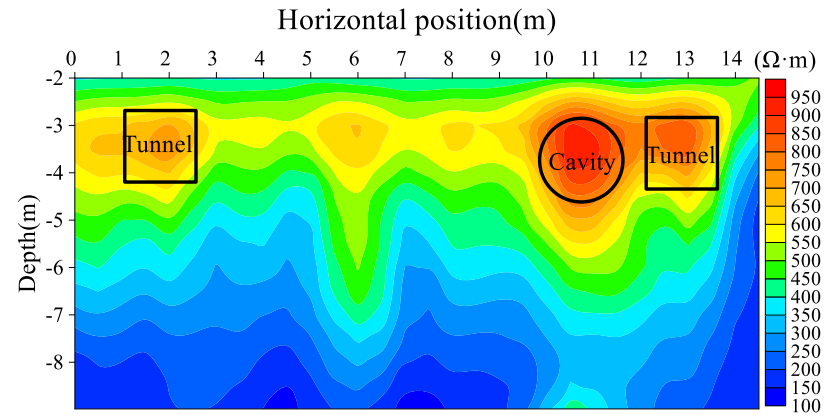

Fig. 8. Apparent resistivity section of physical model.

Compared with models and measuring results, we can conclude that the geological model and measuring result match very well.

\section{CONCLUSIONS}

According to the above presented research work, we can conclude the following:

1. Numerical modelling results show that the response characteristics of transient electromagnetic method of high resistivity goaf are weaker than that of low resistivity goaf. However, the response of high resistivity goaf can still be recognized by simple processing of the received voltage curves.

2. Physical modelling results show that high resistivity anomalous objects can be effectively detected. Therefore, it appears that our research can be useful for the application of transient electromagnetic method (TEM) in the exploration of the earth.

\section{REFERENCES}

[1] Du Kun et al., "Comprehensive evaluation of underground goaf risk and engineering application", Journal of Central South University, vol. 42, no. 9, pp. 2803-2811, 2011.

[2] H. Poor, An Introduction to Signal Detection and Estimation. New York: Springer-Verlag, 1985, ch. 4.

[3] Wang Jilin et al., "Numerical analysis of effect of abandoned goaf foundation deformation on ground buildings", Journal of Mining \& Safety Engineering, vol. 25, no. 4, pp. 477-480, 2008.

[4] Liu Jinghua et al., "The geophysical exploration about exhausted area and sinking area in coal mine", Journal of China Coal Society, vol. 30, no. 6 , pp. 715-719, 2005.

[5] Xu Ping, Mao Xianbiao, Zhang Minxia, Zhou Yuejin, Yu Bangyong. "Safety analysis of building foundations over old goaf under additional stress from building load and seismic actions", International Journal of Mining Science and Technology, vol. 24, no. 5, pp. 713-718, 2014. DOI: 10.1016/j.ijmst.2014.03.030.

[6] Xue Guoqiang et al., "Feasibility analysis of transient electromagnetic method for detectiong underground cave", Journal of the University of Petroleum, vol. 28, no. 5, pp. 135-138, 2004.

[7] Yu Chuantao, Liu Hongfu, Gao Jianping, "The experimental study on the Dual frequency induced polarization method detecting coal mine gob", Progress in Geophysics, vol. 23, no. 5, pp. 1603-1609, 2008.

[8] Chen Weiying, Xue Guoqiang, "Application on coal-mine voids detection with multi-device TEM technology", Progress in Geophysics, vol. 28, no. 5, pp. 2709-2717, 2013.

[9] Fan Tao, "Application of TEM detected goaf in coal mine with highresistivity and shallow layer", Int. Conf. Machine Intelligence (ICMI 2011), 2011.

[10] Xie Haijun, "Research on TEM detection technology for wateraccumlating gob in coal mines", Ph.D. dissertation, China University of Geosciences, Beijing, 2009.

[11] B. Su, J. Yu, C. Sheng, "Maxwell-equations based on mining transient electromagnetic method for coal mine-disaster water 
detection", Elektronika ir Elektrotechnika, vol. 23, no. 3, 2017. DOI: 10.5755/j01.eie.23.3.18326.

[12] Li Jianhui, Zhu Ziqiang, Liu Shucai, "The transient electromagnetic field that excited by rectangular transmitting loop based on Gaver Stehfest algorithm", Oil Geophysical Prospecting, vol. 46, no. 3, pp. 489-492, 2011.

[13] M. N. Nabighian, Ed., Electromagnetic Methods in Applied Geophysics. Society of Exploration Geophysicists, 1991. DOI: 10.1190/1.9781560802686.

[14] J. H. Knight, A. P. Raiche, "Transient electromagnetic calculations using the Gaver-Stehfest inverse Laplace transform method", Geophysics, vol. 47, no. 1, pp. 47-50, 1982. DOI $10.1190 / 1.1441280$

[15] Li Maofei, "Study on the transient electromagnetic interpretation method based on the inversion model of seismic wave impedance", Ph.D. dissertation, China University of Mining and Technology, 2017.

[16] Niu Zhilian, "Principle of time domain electromagnetic method", M.S. thesis, The Publishing House of Central South University, 2007, pp. 22-25. 